\title{
Developmental Regulation of GTP-CH1 in the Porcine Lung and Its Relationship to Pulmonary Vascular Relaxation
}

\author{
MANASI NANDI, JAMES LEIPER, FRANCESCA ARRIGONI, ALISON HISLOP, PATRICK VALLANCE, AND \\ SHEILA HAWORTH \\ Division of Medicine, British Heart Foundation Laboratories, The Rayne Institute, University College London, \\ London, WC1E 6JJ, United Kingdom
}

\begin{abstract}
Nitric oxide (NO) plays an important role in lowering pulmonary vascular resistance after birth. However, in persistent pulmonary hypertension of the newborn (PPHN) NO-mediated dilation is dysfunctional. GTP-cyclohydrolase 1 (GTP-CH1) is the ratelimiting enzyme for the biosynthesis of $6 R$-L-erythro-5,6,7,8tetrahydrobiopterin $\left(\mathrm{BH}_{4}\right)$ an essential cofactor for nitric oxide synthase (NOS) activity. Suboptimal levels of $\mathrm{BH}_{4}$ may result in NOS uncoupling and the subsequent generation of harmful superoxide anions. We therefore investigated the functional effects of supplementing $\mathrm{BH}_{4}$ and/or a superoxide dismutase mimetic (MnTMPyP) in porcine intrapulmonary arteries from normal animals and from a porcine model of PPHN. We investigated whether any functional effects of $\mathrm{BH}_{4}$ could be explained by changes in GTP-CH1 expression. Supplementation of $\mathrm{BH}_{4}$ significantly improved endotheliumdependent relaxations in arteries from 3- and 14-d-old healthy animals, whereas no effect was seen in vessels from younger animals and adults. GTP-CH1 protein expression was lowest at 3 and $14 \mathrm{~d}$, suggesting a rate limitation of $\mathrm{BH}_{4}$ at this time. $\mathrm{BH}_{4}$ supplementation alone did not improve the relaxant response to acetylcholine in arteries obtained in a porcine model of PPHN. Furthermore, GTP$\mathrm{CH} 1$ protein expression was normal for age. However, co-treatment with both $\mathrm{BH}_{4}$ and MnTMPyP restored endothelial function. GTP$\mathrm{CH} 1$ is developmentally regulated in the pulmonary vasculature of neonates and this results in a functionally significant limitation of $\mathrm{BH}_{4}$. Although GTP-CH-1/BH $\mathrm{BH}_{4}$ levels alone do not explain the profound endothelial dysfunction seen in PPHN, increasing NO bioavailability by supplementing $\mathrm{BH}_{4}$ and quenching superoxide may prove to be therapeutically beneficial. (Pediatr Res 59: 767772, 2006)
\end{abstract}

$\mathrm{P}$ ulmonary vascular resistance falls rapidly after birth as the pulmonary vasculature adapts to extrauterine life. Structural remodeling and the maturation of relaxant mechanisms are triggered by factors related to parturition and inhalation of oxygen $(1,2)$, and failure of these processes leads to PPHN (3).

The vascular endothelium plays an important role in regulating vascular smooth muscle tone via the release of several vasoactive products, one of which is nitric oxide (NO) (4) produced from L-arginine and oxygen by nitric oxide synthase (NOS). There is increasing evidence that the NO pathway is

Received September 21, 2005; accepted January 23, 2006.

Correspondence: Manasi Nandi, Ph.D., Division of Medicine, British Heart Foundation Laboratories, The Rayne Institute, University College London, 5 University Street, London WC1E 6JJ, United Kingdom; e-mail: m.nandi@ucl.ac.uk

Supported by the Medical Research Council and the British Heart Foundation.

DOI: 10.1203/01.pdr.0000219301.19958.a0 dysfunctional in PPHN, and treatment of neonates suffering from PPHN with inhaled NO has potential therapeutic benefits. However, the regulation of NO generation during normal adaptation and the mechanisms underlying maladaptation are unclear, particularly as NOS seems to be present but its activity and functional effects are reduced (5). NO generation can be limited by a deficiency of the essential NOS cofactor, $\mathrm{BH}_{4}$. Suboptimal levels of $\mathrm{BH}_{4}$ may result in NOS uncoupling and the subsequent generation of $\mathrm{O}_{2}^{-}(6,7)$. The activity of the enzyme GTP-CH1 is rate limiting for the generation of $\mathrm{BH}_{4}$ (8), and, recently, we and others demonstrated that adult mice deficient in GTP-CH1 have a pulmonary hypertensive phenotype $(9,10)$. The aim of the current study was to investigate whether the GTP-CH1/BH $\mathrm{BH}_{4}$ pathway is developmentally regulated in the perinatal pulmonary vasculature and whether this pathway is dysfunctional in PPHN.

\section{MATERIALS AND METHODS}

Acquisition of tissue samples. Porcine lungs were obtained from Large White piglets, and the following age groups were studied: newborn, $12-24 \mathrm{~h}$, $3 \mathrm{~d}, 14 \mathrm{~d}$, and adult ( $n \geq 4$ per age group). To induce PPHN, naturally delivered newborn piglets were placed in a hypobaric chamber maintained at $50.8 \mathrm{kPa}$ for $3 \mathrm{~d}$ within $20 \mathrm{~min}$ after birth. The chamber, maintained at $29^{\circ} \mathrm{C}$, contained a heating lamp and straw bedding, and piglets were gavage fed on modified cow's milk during this time $(4 \times 30$ min feeds per day). After $3 \mathrm{~d}$ in the chamber, piglets were killed. Piglets placed in a hypobaric chamber develop pulmonary hypertension and characteristically have an arterial oxygen saturation of $71 \pm 5 \%$ and right ventricular hypertrophy compared with age matched controls (11). Piglets removed from their mother and reared under the same conditions (but not exposed to hypobaric hypoxia) were used as controls. The weight ratio of right ventricle to left ventricle plus septum was determined in each animal.

All animals were killed with an overdose of pentobarbitone $(100 \mathrm{mg} / \mathrm{kg})$, with the exception of adult pigs (killed at the abattoir by captive bolt). All heart-lung blocks were removed immediately after death and immersed in chilled Krebs-Henseleit solution $(119 \mathrm{mM} \mathrm{NaCl}, 4.7 \mathrm{mM} \mathrm{KCl}, 25 \mathrm{mM}$ $\mathrm{NaHCO}_{3}, 1.2 \mathrm{mM} \mathrm{MgSO}$; $1.2 \mathrm{mM} \mathrm{KH} \mathrm{PO}_{4}$ and $11 \mathrm{mM}$ glucose plus 2.5 $\mathrm{mM} \mathrm{CaCl} 2$ ) until subsequent use. All animal studies were carried out in compliance with the Animal Procedures Act 1986 under a Home Office License.

Functional measurement of pulmonary vascular reactivity in response to exogenous $\mathrm{BH}_{4}$ supplementation. Axial intrapulmonary arteries were dissected from one upper lobe per animal and cleaned of excess connective

Abbreviations: $\mathbf{B H}_{\mathbf{4}}, 6 R$-L-erythro-5,6,7,8-tetrahydrobiopterin; GTP-CH1, GTP-cyclohydrolase 1; MnTMPyP, Mn (III) tetrakis (1-methyl-4-pyridyl) porphyrin pentachloride (superoxide dismutase mimetic); $\mathbf{O}_{2}{ }^{-}$, superoxide anion; PPHN, persistent pulmonary hypertension of the newborn; SOD, superoxide dismutase 
tissue; care was taken to maintain an intact endothelial layer. Vessels were set up for standard organ chamber pharmacology as previously described (12). Vessels were incubated for 30 min with either the $\mathrm{BH}_{4}$ precursor sepiapterin $(30 \mu \mathrm{M})$ (Schircks Laboratories, Jona, Switzerland), the SOD mimetic MnTMPyP (10 $\mu \mathrm{M})$ (Calbiochem, San Diego, CA), sepiapterin plus MnTMPyP, or Krebs Henseleit alone (vehicle) and contracted with the thromboxane analogue, U46619 (EC80) (Sigma Chemical Co., St. Louis, MO). Although it may be possible that MnTMPyP acts differently than native SOD, MnTMPyP was chosen for this study due to its cell permeability. Initial studies showed that sepiapterin/MnTMPyP administration had no effect on the response to U46619 when compared with control tissue (data not shown). Once a plateau in the contraction to U46619 had been reached, a concentration response curve to acetylcholine (ACh) was constructed ( $1 \mathrm{nM}-0.3 \mathrm{mM})$ after which vessels were washed for $10-15 \mathrm{~min}$ and allowed to return to baseline. Vessels were then reincubated for $30 \mathrm{~min}$ with sepiapterin $(30 \mu \mathrm{M})$ and/or MnTMPyP (10 $\mu \mathrm{M})$ or Krebs Henseleit and recontracted with U46619 (EC80) and a concentration response curve to the NO donor, sodium nitroprusside (SNP, $1 \mathrm{nM}-3 \mu \mathrm{M}$ ) constructed. Finally, $10 \mu \mathrm{M}$ papaverine was added to all vessels to allow the tissue to relax fully.

A selection of vessels were fixed after organ chamber experimentation and embedded in paraffin wax, and 4- $\mu \mathrm{m}$ sections cut. The presence of an intact endothelial layer confirmed using a hematoxylin and eosin stain.

Measurement of GTP-CH1 and eNOS protein expression in lung homogenates. The cardiac lobes from porcine lungs were snap frozen immediately after euthanasia. The lobes were homogenized in Tris buffer $(500 \mathrm{mM}$, $\mathrm{pH}$ 7.5) containing protease inhibitors (Roche Molecular Biochemicals, Mannheim, Germany), 0.1M EDTA, and 0.1 mM EGTA. Samples were separated into soluble and particulate fractions as described previously (5) and the protein concentrations in each fraction determined using a standard Bradford protein assay (Bio-Rad, Hemel Hempstead, UK). Levels of GTP$\mathrm{CH} 1$ or eNOS protein were measured in soluble and particulate fractions, respectively, by standard Western blotting. Briefly, protein samples (80 $\mu \mathrm{g} /$ well) were resolved by electrophoresis through $12 \%$ SDS-PAGE gels for GTP-CH1 and 8\% SDS-PAGE gels for eNOS, electroblotted onto PVDF membranes that were subsequently probed with a 1:1000 dilution of a polyclonal primary GTP-CH1 antipeptide antibody (raised against amino acids 17-45, Sigma Chemical Co.) or a 1:1000 dilution of eNOS antibody (Santa Cruz Biotechnologies, Santa Cruz, CA) in PBS with 1\% Tween (PBST) followed by a 1:3000 dilution of secondary anti-rabbit horseradish peroxidase-conjugated antibody in PBST (Amersham Pharmacia Biotech, Little Chalfont, Buckinghamshire, UK; Santa Cruz Biotechnologies). Signals were developed using ECL reagents (Amersham Pharmacia Biotech) and quantified by densitometry.

Localization of GTP-CH1 in the lung. Standard immunohistochemical staining techniques (13) were performed on $10-\mu \mathrm{m}$ serial sections cut from frozen blocks of porcine cardiac lobe, with the following modifications: sections were blocked with avidin and biotin for 10 min each and a protein blocker (DAKO, Bucks, UK) for $30 \mathrm{~min}$ at room temperature (RT) and sections incubated overnight at $4{ }^{\circ} \mathrm{C}$ with the GTP-CH1 antipeptide antibody diluted 1:250 in PBS containing 4\% normal swine serum. This was followed by four washes in PBS and a 30-min incubation with biotinylated swine anti-rabbit secondary antibody 1:300 (DAKO). Positive immunoreactivity was demonstrated using avidin-biotinylated horseradish peroxidase (Amersham Pharmacia Biotech) and diaminobenzidine (Sigma Chemical Co.). Negative controls were treated in the same way except the primary GTP-CH1 antibody was omitted.

Data analysis. Relaxation to ACh or SNP is expressed as percentage decreases in tension from the maximum contraction induced by U46619 to the maximum relaxation induced by papaverine. Concentration response curves were compared with two-way ANOVA with Bonferroni post hoc test. All other statistical analysis was carried out using ANOVA or $t$ test where appropriate.

\section{RESULTS}

Contractile and relaxant effects in normal porcine pulmonary arteries. Initial studies showed that preincubation with the NOS inhibitor $\mathrm{N}_{\omega}$-nitro-L-arginine (L-NAME, $1 \mathrm{mM}$ ) abolished ACh-mediated relaxations in porcine pulmonary arteries (data not shown), demonstrating that ACh-mediated relaxations were NOS dependent.

Functional studies performed on conduit pulmonary arteries obtained from healthy pigs revealed an age-dependent increase in endothelium-dependent relaxations to $\mathrm{ACh}$ [Fig. 1a(ii)] with no significant differences in the response to SNP (Fig. 1b).

ACh responses were significantly higher in 3- and 14-d-old and adult animals compared with the newborn and 12-24 h age groups and correlated with the expression of eNOS protein [Fig. $1 a(i)$ ].

In vitro effects of sepiapterin during normal development. U46619 caused a sustained and stable contractile response in all vessels that was unaltered by incubation with sepiapterin and/or MnTMPyP. Sepiapterin had no effect on the maximum endothelium-dependent relaxations to ACh in newborn, 12-24 $\mathrm{h}$, and adult vessels, but at 3 and $14 \mathrm{~d}$ of age, sepiapterin significantly enhanced the maximum endothelium dependent relaxations to ACh (Fig. 2a), the same temporal pattern was observed in vessels incubated with MnTMPyP alone (Fig. $2 b$ ). In view of these findings, a further study was conducted to investigate the effects of co-incubating sepiapterin $(30 \mu \mathrm{M})$ and the superoxide dismutase mimetic MnTMPyP $(10 \mu \mathrm{M})$ to determine whether the effects of sepiapterin could be enhanced. There was no statistically significant difference in the maximum response to $\mathrm{ACh}$ between vessels treated with either sepiapterin alone or those co-incubated with both sepiapterin and MnTMPyP (Fig. 2, $c$ and $d$ ). Endotheliumindependent relaxations to SNP were unaffected by either sepiapterin and/or MnTMPyP (Fig. 2e).

In vitro effects of sepiapterin in pulmonary hypertensive piglets. The weight ratios of the right ventricle to left ventricle + septum were higher in piglets exposed to $3 \mathrm{~d}$ of hypobaric

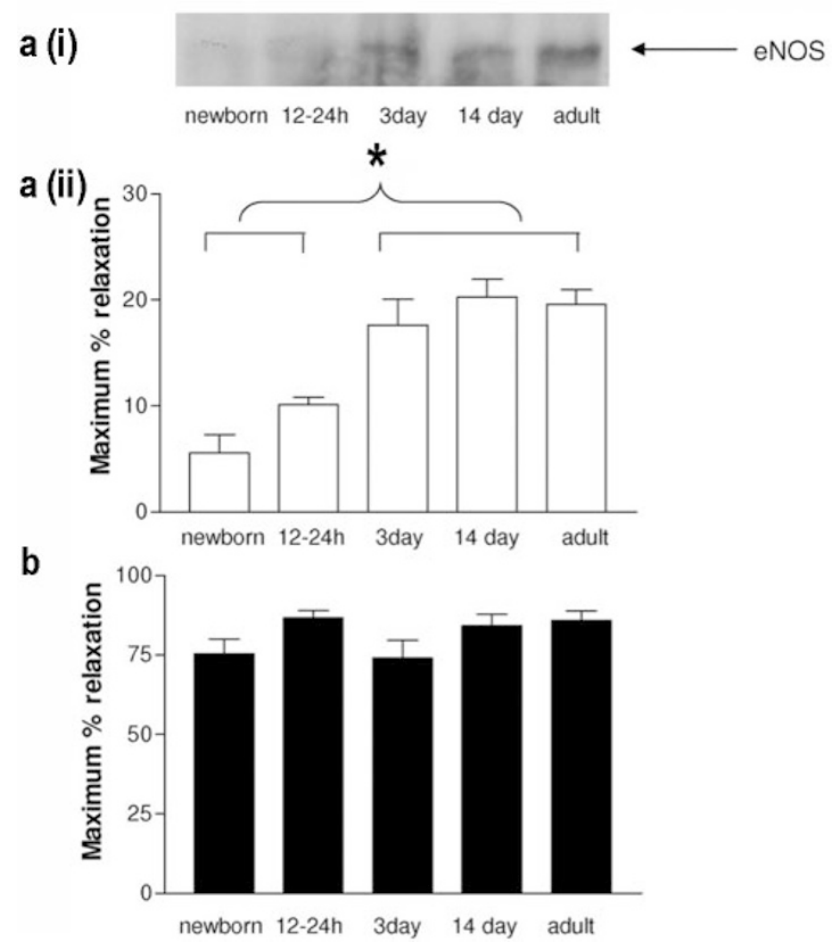

Figure 1. (a) i) Immunoblot showing temporal expression of eNOS protein in the particulate fraction of porcine lung homogenates; ii) maximum relaxation to ACh in untreated porcine pulmonary arteries $(n \geq 4, * p<0.05)$. (b) Maximum relaxation to SNP in untreated porcine pulmonary arteries $(n \geq 4)$. 

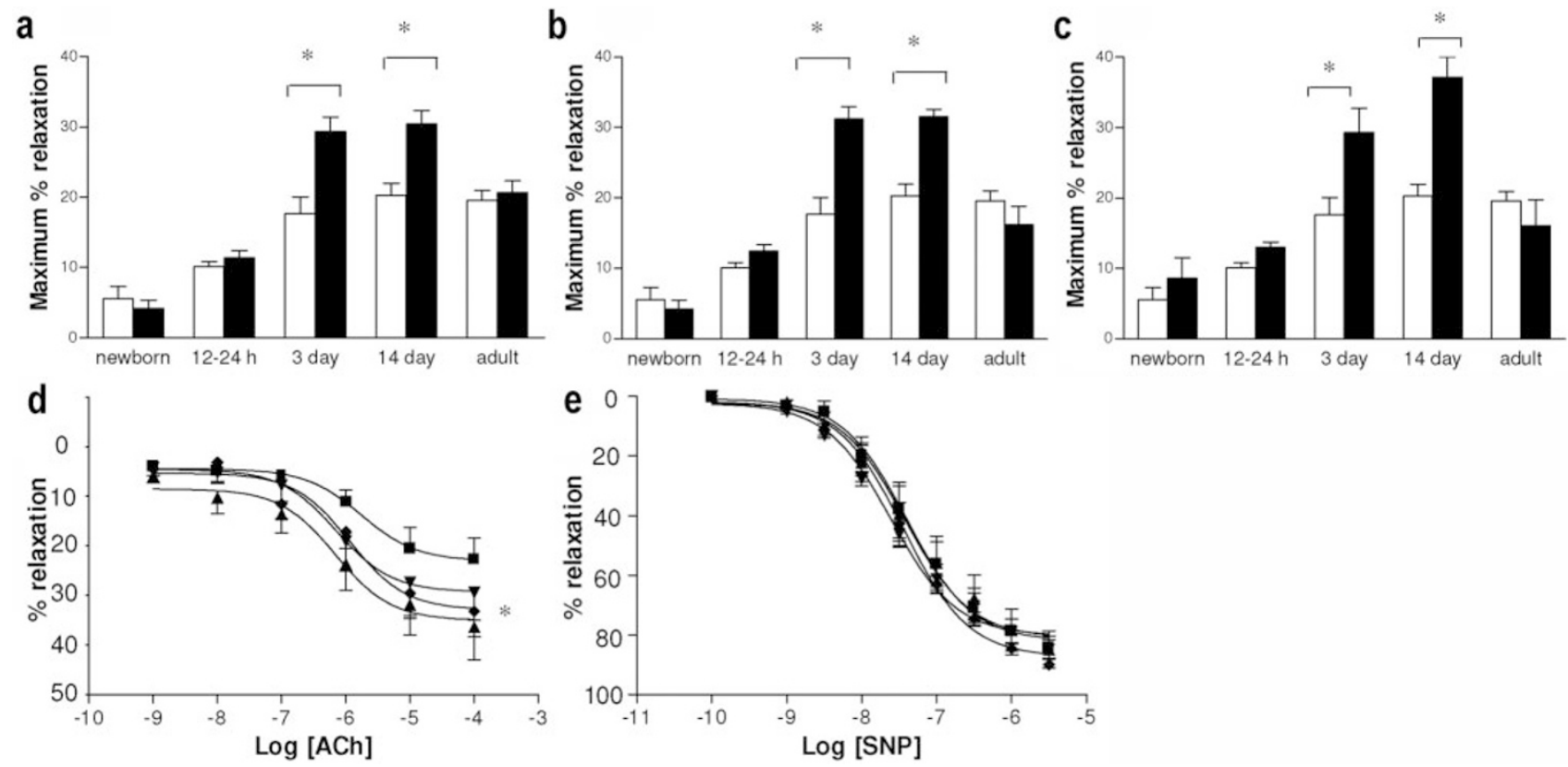

Figure 2. (a) Maximum relaxation to ACh in porcine pulmonary arteries treated with sepiapterin (30 $\mu \mathrm{M})(\square)$ compared with vehicle (Krebs) $(\square)$. $n \geq 4$; * $p$ $<0.05$ (sepiapterin $v s$ vehicle for each age group). (b) Maximum relaxation to ACh in porcine pulmonary arteries treated with MnTMPyP $(10 \mu \mathrm{M})(\mathbf{\square})$ compared with vehicle (Krebs) $(\square) . n \geq 4 ; * p<0.05$ (MnTMPyP $v s$ vehicle for each age group). (c) Maximum relaxation to ACh in porcine pulmonary arteries treated with a combination of sepiapterin $(30 \mu \mathrm{M})$ and MnTMPyP $(10 \mu \mathrm{M})(\square)$ compared with vehicle (Krebs) $(\square)$. $n \geq 4$; ${ }^{*} p<0.05$ (sepiapterin plus MnTMPyP $v s$ vehicle for each age group). (d) Concentration response curves to ACh in porcine pulmonary arteries obtained from 14-d-old piglets when treated with sepiapterin $(30 \mu \mathrm{M})(\diamond)$, MnTMPyP $(10 \mu \mathrm{M})(\boldsymbol{\nabla})$, and sepiapterin plus MnTMPyP $(\mathbf{\Delta})$ compared with vehicle $($ Krebs $)(\boldsymbol{\square}) . n=5 ; p<0.05$. (e) Concentration response curves to SNP in porcine pulmonary arteries from 14-d-old piglets after treatment with sepiapterin $(30 \mu \mathrm{M})(\diamond), \mathrm{MnTMPyP}(10 \mu \mathrm{M})(\mathbf{\nabla})$, sepiapterin plus MnTMPyP $(\mathbf{\Delta})$, or vehicle (Krebs) $(\boldsymbol{\square}) . n=5$.

hypoxia when compared with age-matched controls and were similar to those in newborn animals (Fig. $3 a$ ), confirming the presence of pulmonary hypertension.
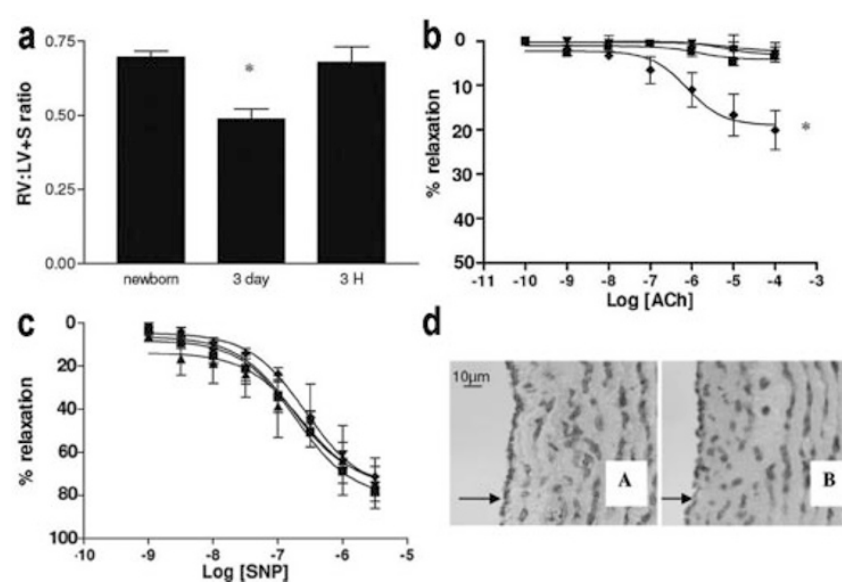

d

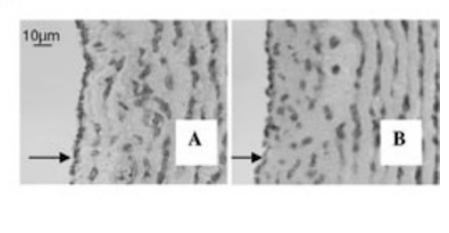

Figure 3. (a) Ratio of right ventricle to left ventricle plus septum $(\mathrm{RV}: \mathrm{LV}+\mathrm{S})$ in newborn, $3 \mathrm{~d}$ control, and after $3 \mathrm{~d}$ hypobaric exposure $(3 \mathrm{H})$. $n \geq 5$; $* p<0.05$. (b) Concentration response curve to $\mathrm{ACh}$ in the presence of sepiapterin $(30 \mu \mathrm{M})(\mathbf{\Delta})$, MnTMPyP $(10 \mu \mathrm{M})(\boldsymbol{\nabla})$, sepiapterin + MnTMPyP $(\checkmark)$, or vehicle (匹), in conduit pulmonary arteries from 3-d-old piglets with hypoxia-induced pulmonary hypertension. $n=6$; * $p<0.05$ (drug-treated $v s$ vehicle). (c) Concentration response curve to SNP in the presence of sepiapterin $(30 \mu \mathrm{M})(\mathbf{\Lambda})$, $\operatorname{MnTMPyP}(\boldsymbol{\nabla})$, sepiapterin plus $\operatorname{MnTMPyP}(\bullet)$, or vehicle ( $\mathbf{\square})$ in conduit pulmonary arteries from 3-d-old piglets with hypoxia-induced pulmonary hypertension. $n=6$. (d) Hematoxylin and eosin staining on arterial rings from 3-d-old control $(A)$ and 3-d-old pulmonary hypertensive $(B)$ after organ chamber experiment showing presence of intact endothelium (marked by arrows) ( $\times 40$ objective).
In the 3-d-old piglets with pulmonary hypertension, relaxation to ACh was absent, consistent with published findings (12), and addition of sepiapterin or MnTMPyP alone did not improve relaxation (Fig. 3b). However, co-incubation of sepiapterin and MnTMPyP significantly improved endotheliumdependent relaxation (Fig. 3b). Endothelium-independent relaxation to SNP was normal and did not differ from that seen in the 3-d-old normoxic animals (Fig. 3c). Tissue obtained from piglets reared in the same way as pulmonary hypertensive piglets (with the exception of hypobaric hypoxia) responded in the same way as a 3-d-old normal control animal (data not shown), demonstrating that the functional effects observed in the 3-d-old pulmonary hypertensive piglets were due to hypobaric hypoxic exposure alone and not as a result of the changed diet or stress caused by separation from the mother. Hematoxylin and eosin staining of a selection of rings after experimentation revealed the presence of an intact endothelial layer in these vessels indistinguishable from that seen in age-matched controls, suggesting the lack of relaxation to $\mathrm{ACh}$ was not due to endothelial damage incurred during experimentation (Fig. 3d).

GTP-CH1 protein expression during normal development. An antipeptide antibody raised against a human GTP-CH1 peptide sequence detected a band of the expected size (28-30 $\mathrm{kD}$ ) in the soluble lung homogenates from all groups studied [Fig. 4a(i and ii)]. GTP-CH1 expression was high in newborn animals and in 12-24 $\mathrm{h}$ age groups significantly lower at 3 and $14 \mathrm{~d}$ of age, with an increased level of expression in the adult [Fig. 4a). By expressing the effect of sepiapterin on the 


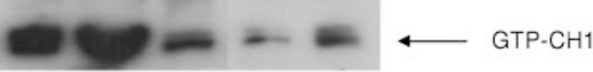

newborn $12-24$ h 3 day 14 day Adult

$4 \mathrm{a}$ (ii)

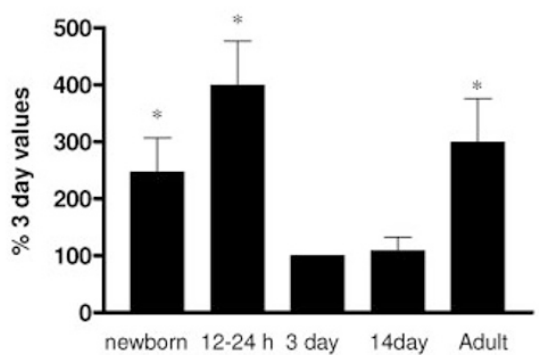

$4 b$

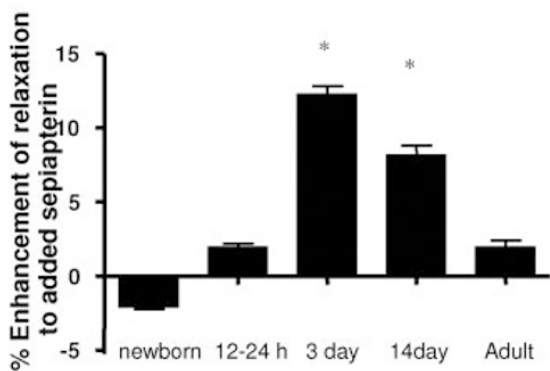

Figure 4. (a) GTP-CH1 protein expression in porcine-soluble lung homogenates. i) Immunoblot showing immunoreactive band at $28-30 \mathrm{kD}$ for all age groups. ii) Densitometric analysis of immunoblots $(n=6)$; all values calculated as a percentage of $3-\mathrm{d}$ values. Values represent mean $\pm \mathrm{SEM} ;{ }^{*} p<0.05$ GTP-CH1 protein expression significantly different from 3-d values. (b) Developmental change in percentage enhancement of maximum relaxation to ACh over control values in sepiapterin-treated vessels. $n \geq 5$; ${ }^{*} p<0.05$.

ACh-mediated relaxation as a percentage enhancement of relaxation over vehicle, it can be seen that where sepiapterin has an effect, GTP-CH1 protein is lowest, suggesting that GTP-CH1 expression might be limiting for $\mathrm{BH}_{4}$ availability at 3 and $14 \mathrm{~d}$ of age (Fig. $4 b$ ).

GTP-CH1 protein expression in pulmonary hypertensive piglets. A comparison of the GTP-CH1 protein expression between 3-d-old pulmonary hypertensive and 3-d-old normal piglets showed that there was no significant difference between the two groups (Fig. 5, $i$ and ii).

Localization of GTP-Ch1. Immunohistochemical staining using the GTP-CH1 antipeptide antibody revealed that GTP$\mathrm{CH} 1$ was localized to the endothelial cells of conduit pulmonary arteries, veins, airway epithelium, and nerves (Fig. 6). This distribution was unchanged with age or with pulmonary hypertension.

\section{DISCUSSION}

We have demonstrated that endothelium-dependent relaxations of porcine conduit pulmonary arteries are low immediately after birth and increase thereafter, reaching a peak in the first $2 \mathrm{wk}$ of life. These findings are consistent with previous observations $(12,14)$. The new findings in this study are that expression of GTP-CH1 in the lungs changes significantly in
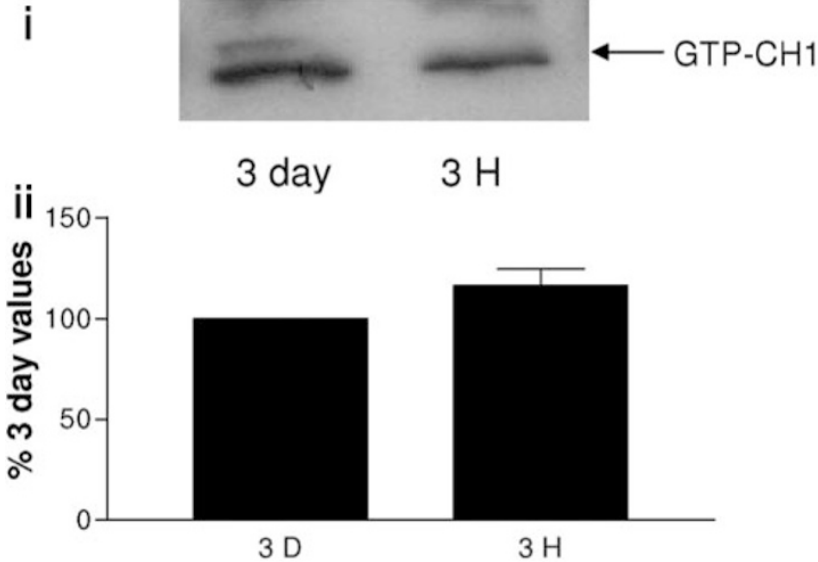

Figure 5. i) Immunoblot showing immunoreactive band $28-30 \mathrm{kD}$ for GTP-CH1 protein expression in porcine-soluble lung homogenates from 3-d control and 3-d pulmonary hypertensive $(3 \mathrm{H})$ piglets. ii) Densitometric analysis of immunoblots; 3 -d pulmonary hypertensive calculated as a percentage of 3 d. $n=6$.
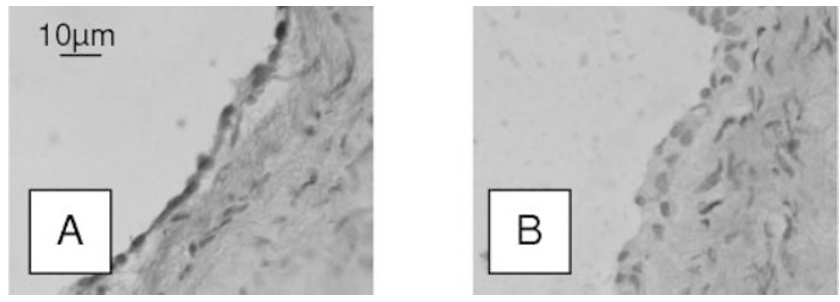

Figure 6. Immunohistochemical staining of porcine lung sections. Positive GTP-CH1 staining seen in endothelial cells of conduit pulmonary artery $(A)$ and negative (no primary antibody) $(B)$ from 12 - to 24 -h-old animal $(\times 40$ objective).

the first few days of life and that supplementation with sepiapterin enhances endothelium-dependent relaxation, suggesting that a functional deficit of $\mathrm{BH}_{4}$ limits endotheliumdependent responses. Although we did not find an improvement of endothelium-dependent relaxations with sepiapterin alone in our model of pulmonary hypertension, the combination of sepiapterin together with a SOD mimetic enhanced relaxation. These findings support recent observations in mice that GTP-CH1 deficiency can cause pulmonary hypertension, are consistent with the finding that increased $\mathrm{O}_{2}{ }^{-}$ contributes to the effects of GTP-CH1 deficiency (10), and identify significant early developmental changes in GTP-CH1 expression.

Normal development. Sepiapterin and/or MnTMPyP had no effect on endothelium-dependent relaxations in newborn, 12- to 24-h-old, and adult porcine pulmonary arteries yet significantly improved relaxations in pulmonary arteries from 3- and 14-d-old pigs. The endothelium-independent relaxations to SNP remained unchanged in all groups, confirming that the effects of sepiapterin were endothelium dependent. These functional findings imply that $\mathrm{BH}_{4}$ becomes rate limiting for $\mathrm{NO}$ generation at 3 and $14 \mathrm{~d}$ but is not rate limiting in newborn and $12-24 \mathrm{~h}$ or adult age groups. Interestingly, at the time GTP-CH1 expression decreases dramatically (d 3) from 
the high levels at birth, eNOS expression and activity have already increased from the low levels seen at birth. The results suggest that this temporary mismatch between GTP-CH1 and NOS expression levels results in a functional deficit of $\mathrm{BH}_{4}$. The lack of ACh relaxation in the newborn age groups may possibly occur as a result of low basal generation of NO coupled with an excess of vasoconstrictors as contractions to U46619 have been shown to be higher in these age groups (12).

The finding that MnTMPyP also improves endotheliumdependent relaxation in these vessels and that the effects of sepiapterin and MnTMPyP are not additive is consistent with enhanced $\mathrm{O}_{2}{ }^{-}$generation, contributing to the effects of $\mathrm{BH}_{4}$ deficiency. Indeed, in isolated enzyme systems, $\mathrm{BH}_{4}$-deficient NOS may generate $\mathrm{O}_{2}{ }^{-}$. It is also possible that there are changes in endogenous SOD levels during development, and these may also contribute the effects we observe with MnTMPyP.

One limitation of this study was that GTP-CH1 protein was measured in whole lung homogenates rather than in isolated intrapulmonary arteries. However, immunohistochemistry confirmed that GTP-CH1 protein was present in the endothelial cell layer of large pulmonary arteries and in nerves, and this pattern appeared unchanged with age. Furthermore, there was a clear relationship between the GTP-CH1 expression in total lung homogenate and the relaxant response to supplemented $\mathrm{BH}_{4}$, and, together, these changes are consistent with a change in $\mathrm{BH}_{4}$ in the vessel. It is not clear why GTP-CH1 expression should alter so markedly in the first few days of life, but one explanation may be the existence of three putative shear stress response elements in the GTP-CH1 promoter sequence. It is possible that the sudden increase in shear stress in the pulmonary vasculature during parturition induces GTP$\mathrm{CH} 1$ expression and the subsequent decrease in this expression at days 3 and 14 may result from a reduction in shear stress following the natural adaptation to air breathing. Further studies would be needed to test this and other explanations are possible. It would also be interesting to know whether developmental changes in GTP-CH1 are also seen in other tissues.

Pulmonary hypertension. Alterations in NO signaling have been shown to contribute to or be associated with the development of pulmonary hypertension in a number of different models (15-18), and mice deficient in $\mathrm{BH}_{4}$ have a pulmonary hypertensive phenotype $(9,10)$. As GTP-CH1 expression was low and $\mathrm{BH}_{4}$ supplementation improved relaxation in 3-d-old healthy animals, we investigated whether loss of GTP-CH1/ $\mathrm{BH}_{4}$ may contribute to the very impaired endotheliumdependent relaxation seen in our 3-d-old model of PPHN.

Endothelium-dependent relaxations to ACh in pulmonary arteries from the 3-d-old PPHN model were absent, whereas endothelium-independent relaxations to SNP remained intact, consistent with previous findings (12). The absence of any beneficial effect of sepiapterin in pulmonary hypertension has been demonstrated previously (19). However, this study demonstrated that combined treatment with sepiapterin and the superoxide dismutase mimetic MnTMPyP improves endothelium-dependent relaxations in this pulmonary hypertensive model. It is interesting that, whereas sepiapterin alone enhances endothelium-dependent relaxation in normal vessels at this stage, in the pulmonary hypertensive vessels addition of the superoxide dismutase mimetic (MnTMPyP) was also required. This is consistent with the critical importance of $\mathrm{O}_{2}{ }^{-}$ identified in the pulmonary hypertension seen in the GTPCH1-deficient mouse (10). GTP-CH1 overexpression results in elevated basal $\mathrm{BH}_{4}$ levels and reduced $\mathrm{O}_{2}{ }^{-}$and protects against hypoxia-induced pulmonary hypertension. Conversely, $\mathrm{BH}_{4}$-deficient mice have increased $\mathrm{O}_{2}{ }^{-}$generation and a pulmonary hypertensive phenotype under normoxic conditions (10). In the present study, we have demonstrated that supplementation of $\mathrm{BH}_{4}$ and simultaneous quenching of $\mathrm{O}_{2}{ }^{-}$restores endothelial function in animals with PPHN. Currently, it is not clear why $\mathrm{O}_{2}{ }^{-}$becomes such an important determinant of responses to $\mathrm{BH}_{4}$ deficiency in pulmonary hypertensive animals but not normal animals. It is of course likely that other NO-independent factor are also involved in the pathogenesis of pulmonary hypertension (20).

In summary, marked developmental regulation of GTP$\mathrm{CH} 1$ occurs during the perinatal period in the porcine lung, resulting in a functionally significant limitation of $\mathrm{BH}_{4}$ for vascular relaxation. Although, in this model of PPHN, the expression levels of GTP-CH1 change in a similar way to the changes seen in a normal animal, it is clear that the functional $\mathrm{BH}_{4}$ deficiency coupled with increased $\mathrm{O}_{2}{ }^{-}$generation contributes to the absent endothelium-dependent relaxations. GTP-CH1 is emerging as a critical regulator of pulmonary vascular tone.

\section{REFERENCES}

1. Haworth SG, Hislop AA 1981 Adaptation of the pulmonary circulation to extrauterine life in the pig and its relevance to the human infant. Cardiovasc Res 15:108-119

2. Heymann MA 1999 Control of the pulmonary circulation in the fetus and during the transitional period to air breathing. Eur J Obstet Gynecol Reprod Biol 84:127-132

3. Abman SH 1999 Abnormal vasoreactivity in the pathophysiology of persistent pulmonary hypertension of the newborn. Pediatr Res 20:103-109

4. Palmer RM, Ferrige AG, Moncada S 1987 Nitric oxide release accounts for the biological activity of endothelium-derived relaxing factor. Nature 327:524-526

5. Arrigoni FI, Hislop AA, Pollock JS, Haworth SG, Mitchell JA 2002 Birth upregulates nitric oxide synthase activity in the porcine lung. Life Sci 70:1609-1620

6. Cosentino F, Patton S, d'Uscio LV, Werner ER, Werner-Felmayer G, Moreau P, Malinski T, Luscher TF 1998 Tetrahydrobiopterin alters superoxide and nitric oxide release in prehypertensive rats. J Clin Invest 101:1530-1537

7. Channon KM 2004 Tetrahydrobiopterin: regulator of endothelial nitric oxide synthase in vascular disease. Trends Cardiovasc Med 14:323-327

8. Nichol CA, Smith GK, Duch DS 1985 Biosynthesis and metabolism of tetrahydrobiopterin and molybdopterin. Annu Rev Biochem 54:729-764

9. Nandi M, Miller A, Stidwill R, Jacques TS, Lam AA, Haworth S, Heales S, Vallance P 2005 Pulmonary hypertension in a GTP-cyclohydrolase 1-deficient mouse. Circulation 111:2086-2090

10. Khoo JP, Zhao L, Alp NJ, Bendall JK, Nicoli T, Rockett K, Wilkins MR, Channon KM 2005 Pivotal role for endothelial tetrahydrobiopterin in pulmonary hypertension. Circulation 111:2126-2133

11. Tulloh RM, Hislop AA, Boels PJ, Deutsch J, Haworth SG 1997 Chronic hypoxia inhibits postnatal maturation of porcine intrapulmonary artery relaxation. Am J Physiol 272:H2436-H2445

12. Arrigoni FI, Hislop AA, Haworth SG, Mitchell JA 1999 Newborn intrapulmonary veins are more reactive than arteries in normal and hypertensive piglets. Am J Physiol 277:L887-L892

13. Hislop AA, Springall DR, Oliveira H, Pollock JS, Polak JM, Haworth SG 1997 Endothelial nitric oxide synthase in hypoxic newborn porcine pulmonary vessels. Arch Dis Child Fetal Neonatal Ed 77:F16-F22

14. Liu SF, Hislop AA, Haworth SG, Barnes PJ Developmental changes in endotheliumdependent pulmonary vasodilatation in pigs. Br J Pharmacol 1992;106:324-330 
15. Giaid A, Saleh D 1995 Reduced expression of endothelial nitric oxide synthase in the lungs of patients with pulmonary hypertension. N Engl J Med 333:214221

16. Champion HC, Bivalacqua TJ, Greenberg SS, Giles TD, Hyman AL, Kadowitz PJ 2002 Adenoviral gene transfer of endothelial nitric-oxide synthase (eNOS) partially restores normal pulmonary arterial pressure in eNOS-deficient mice. Proc Natl Acad Sci U S A 99:13248-13253

17. Fagan KA, Fouty BW, Tyler RC, Morris KG Jr, Hepler LK, Sato K, LeCras TD, Abman SH, Weinberger HD, Huang PL, McMurtry IF, Rodman DM 1999 The pulmonary circulation of homozygous or heterozygous eNOS-null mice is hyperresponsive to mild hypoxia. J Clin Invest 103:291-299
18. Fike CD, Kaplowitz MR, Thomas CJ, Nelin LD 1998 Chronic hypoxia decreases nitric oxide production and endothelial nitric oxide synthase in newborn pig lungs. Am J Physiol 274:L517-L526

19. Steinhorn RH, Russell JA, Lakshminrusimha S, Gugino SF, Black SM, Fineman JR 2001 Altered endothelium-dependent relaxations in lambs with high pulmonary blood flow and pulmonary hypertension. Am J Physiol Heart Circ Physio 280:H311-H317

20. Humbert M, Morrell NW, Archer SL, Stenmark KR, MacLean MR, Lang IM, Christman BW, Weir EK, Eickelberg O, Voelkel NF, Rabinovitch M 2004 Cellular and molecular pathobiology of pulmonary arterial hypertension. J Am Coll Cardiol. 43:13S-24S 